\title{
Crystal Structure of Rat Liver Betaine Homocysteine S-Methyltransferase Reveals New Oligomerization Features and Conformational Changes Upon Substrate Binding
}

\section{Beatriz González', María A. Pajares², Martín Martínez-Ripoll' Tom L. Blundell ${ }^{3}$ and Julia Sanz-Aparicio ${ }^{1 *}$}

\author{
${ }^{1}$ Grupo de Cristalografía \\ Macromolecular y Biología \\ Estructural, Instituto de \\ Química-Física "Rocasolano" \\ CSIC, Serrano 119, 28006 \\ Madrid, Spain
}

${ }^{2}$ Instituto de Investigaciones Biomédicas "Alberto Sols" CSIC-UAM, Arturo Duperier 4, 28029 Madrid, Spain

${ }^{3}$ Department of Biochemistry University of Cambridge Tennis Court Road, Cambridge CB2 1QW, United Kingdom

\begin{abstract}
Betaine homocysteine $S$-methyltransferase (BHMT) is one of the two enzymes known to methylate homocysteine to generate methionine in the liver. It presents a $\mathrm{Zn}^{2+}$ atom linked to three essential Cys residues. The crystal structure of rat liver BHMT has been solved at $2.5 \AA$ resolution, using crystals with $P 2_{1}$ symmetry and $45 \%$ solvent content in the cell. The asymmetric unit contains the whole functional tetramer showing point symmetry 222. The overall fold of the subunit consists mostly of a $(\alpha / \beta)_{8}$ barrel, as for human BHMT. From the end of the barrel, the polypeptide chain extends away and makes many interactions with a different subunit, forming tight dimers. The most remarkable structural feature of rat liver BHMT is the presence of a helix including residues 381-407, at the $C$ terminus of the chain, which bind together the dimers $\mathrm{AB}$ to $\mathrm{CD}$. A strong ion-pair and more than 60 hydrophobic interactions keep this helix stacked to the segment 316-349 from the opposite subunit. Moreover, the crystal structure of free rat liver BHMT clearly shows that Tyr160 is the fourth ligand coordinated to $\mathrm{Zn}$, which is replaced by Hcy upon binding. Two residues essential for substrate recognition, Phe76 and Tyr77, are provided by a conformational change in a partially disordered loop (L2). The crucial role of these residues is highlighted by site-directed mutagenesis.
\end{abstract}

(C) 2004 Elsevier Ltd. All rights reserved.

Keywords: betaine homocysteine S-methyltransferase; Zn-metalloenzyme; $\mathrm{Zn}$ ligands; X-ray structure; subunit interactions

\section{Introduction}

Betaine homocysteine S-methyltransferase (BHMT, EC 2.1.1.5) is one of the two enzymes known to methylate homocysteine (Hcy) to generate methionine in the liver. ${ }^{1}$ This enzyme has been known for a number of years, but only recently, and due to the role of Hcy as a potential independent risk factor for cardiovascular

Abbreviations used: BHMT, betaine homocysteine $S$-methyltransferase from rat liver; h-BHMT, human betaine homocysteine S-methyltransferase; Hcy, homocysteine; B12-MS, B12-dependent methionine synthase; CB-Hcy, S(ס-carboxybutyl)-L-homocysteine; DTT, dithiothreitol; Ada, adenosine deaminase.

E-mail address of the corresponding author: xjulia@iqfr.csic.es diseases, ${ }^{2,3}$ has become a focus of interest. This cytosolic enzyme uses betaine as the methyl donor and renders dimethylglycine as a side product. ${ }^{4}$ Betaine is found naturally in most living organisms, where it acts as an osmolyte..$^{5}$ Synthesis of betaine occurs through choline oxidation, and it can also be obtained externally from food. ${ }^{6}$ Its use in the methylation of Hcy contributes to the reduction of the levels of this amino acid, and allows the recovery of one of the three methylation equivalents used for choline synthesis. The BHMT reaction has been shown to occur mainly in liver and kidney, where the enzyme has been localized by immunohistochemistry, but its apparition in lens has also been reported in Rhesus monkey. ${ }^{8}$ BHMT has been identified associated to tubulin in microtubules $^{9}$ and linked to autophagosomes. ${ }^{10}$ Moreover, it has recently been shown that 
BHMT regulates the expression of apolipoprotein $\mathrm{B}$, leading to an increase in related lipoproteins. ${ }^{11}$

BHMT is an oligomer composed of four identical subunits with 407 amino acid residues in rat. ${ }^{12-15}$ Conservation among mammalian enzymes is very high, the similarity being $80 \%$ and $90 \%$ at the nucleotide and amino acid levels, respectively. ${ }^{11}$ The protein has been shown to contain $\mathrm{Zn}^{2+}, 16$ linked to three cysteine residues (217, 299 and 300 according to the human sequence), ${ }^{17}$ which are contained in two typical motifs for thiol-seleniol methyltransferases, ${ }^{214} \mathrm{GVNCH}^{218}$ and ${ }^{293}$ VRYIGGC $\mathrm{CGFEPYHI}^{307}$. These sequences are preserved in all the species studied: rat, mouse, pig and human. ${ }^{18}$ It has also been shown that glycine residues in these motifs play an important role. Thus, Gly214 is an essential residue, since no mutation at this amino acid is allowed without a complete activity loss. ${ }^{19}$ Moreover, other Gly residues only allow minor changes, as is the case for residues 297, 298 or 301. In addition, residues 10-320 present certain sequence homology to other members of the thiol-seleniol methyltransferases family, which include the $\mathrm{N}$-terminal region of B12-dependent methionine synthase (B12-MS), the other enzyme producing Met from Hcy in mammals, $S$-methylmethionine (or $S$-adenosylmethionine) homocysteine methyltransferases and $S$-methylmethionine (or $S$-adenosylmethionine) selenocysteine methyltransferases. ${ }^{20}$ Although these proteins take the methyl from different molecules, all of them use Hcy, Se-Hcy, and SeCys as methyl acceptors, and must therefore share common substrate binding and enzymatic mechanisms.

The first three-dimensional structure for a member of the family, human BHMT (h-BHMT), has recently been reported on two different forms. ${ }^{21}$ One of them presents the protein in an oxidized state without $\mathrm{Zn}^{2+}$ and includes a disulphide bond between Cys217 and Cys299, two of the ligands identified for this metal site. ${ }^{18}$ The second structure available presents a reduced form of BHMT including the cation and the transition state analogue $S$-( $\delta$-carboxybutyl)-Lhomocysteine (CB-Hcy). However, there are several unordered regions that remain unsolved. In particular, a long region of more than 30 residues at the C-terminal end of the polypeptide chain is not visible. The purpose of this work is to get insight into the structure of free native rat liver BHMT and to clarify the unsolved areas of the protein structure, the high degree of homology among human and rat proteins allowing the immediate extrapolation of the data.

\section{Results and Discussion}

\section{Structure determination and the model}

The crystal structure of BHMT from rat liver has been solved at $2.5 \AA$ resolution. The purification and crystallization of the recombinant protein, as well as data collection for native, heavy-atom derivatives and the SeMet-modified form have been reported by us. ${ }^{15}$ The structure determination of the human enzyme ${ }^{21}$ allowed the use of molecular replacement by using the coordinates of the human monomer as a model. Experimental details and structure determination procedures are given in Materials and Methods and in Table 2. The rat liver crystals belong to the $P 2_{1}$ space group, with $45 \%$ solvent content in the cell. The asymmetric unit contains the whole functional tetramer showing point symmetry 222 that is shown in Figure 1. Each BHMT subunit consists of 407 residues, which are included in the model except for two regions that are not visible in the electron density maps. The first of these regions is the N-terminal Met1-Ala9. The second region is loop L2 connecting $\beta 2$ to $\alpha 2$ of the barrel, which presents a great mobility crucial for substrate binding, as shown below. Hence, residues Lys82-Val90 are disordered in the more complete subunit, whereas this region extends from Ser79 to Ile94 in the less complete molecule.

The overall fold of the subunit can be observed in Figure 2(a) and consists mostly of an $(\alpha / \beta)_{8}$ barrel with additional units of secondary structure inserted between the $\alpha / \beta$ (residues 10-318), an extended arm including residues $319-370$ and mainly involved in dimerization contacts, a flexible linker from 371 to 380 , and a long $\alpha$-helix including residues 381-406. From the inspection of the initial electron-density maps, it became obvious that the orientation of this C-terminal helix was different in the four subunits. Consequently, the model was refined imposing non-crystallographic symmetry restrains between the four molecules of the asymmetric unit in two separate blocks: residues 10-370 forming the barrel and the extended arm (RMSD $0.23 \AA$ ) and residues $381-406$ of the long C-terminal helix (RMSD $0.53 \AA$ ). As it can be seen in Figure 2(b), where the barrels from the four subunits are superimposed, the L1 (residues 38-52) and the L7 (residues 248-277) loops lie over the C-terminal face of the barrel and shape the

Table 1. BHMT activity of mutant proteins

\begin{tabular}{lc}
\hline & BHMT activity (nmol/min per $\mathrm{mg}$ ) \\
\hline Wild-type & $17.07 \pm 0.12$ \\
Y160F & $0.11 \pm 0.07$ \\
Y160T & $0.53 \pm 0.09$ \\
Y160A & $0.92 \pm 0.19$ \\
F76A & $0.14 \pm 0.05$ \\
F76D & $0.28 \pm 0.02$ \\
\hline
\end{tabular}

Mutants for residues Y160 and F76 of rat liver BHMT were prepared as described (see Materials and Methods). After expression and purification the activity of the mutant proteins was assayed according to the radio assay described. ${ }^{38}$ The results included in the Table are the mean \pm SD of a typical experiment. 

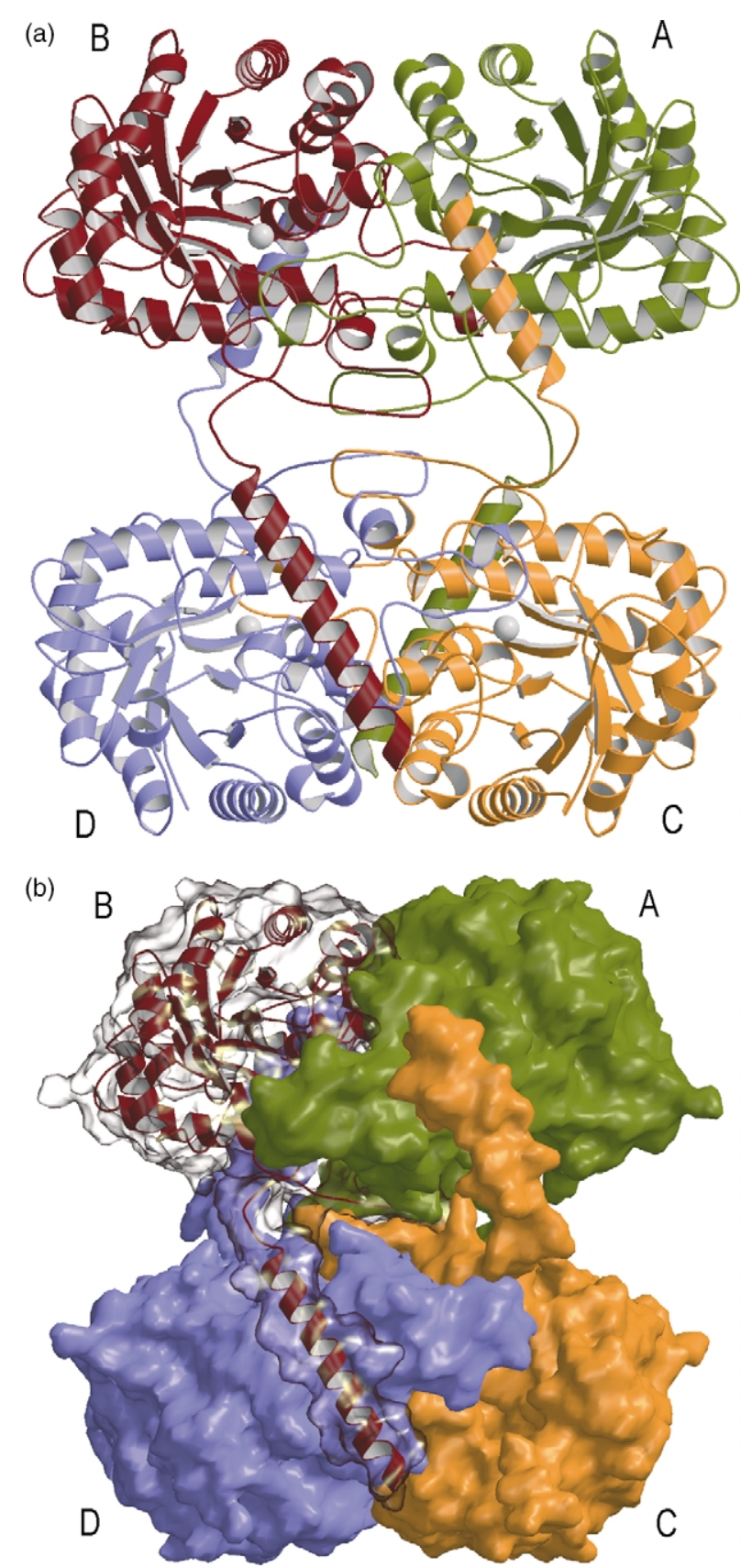

Figure 1. Structure of the rat liver BHMT tetramer. (a) The asymmetric unit contains four molecules related by local 222 symmetry, which are represented in a different colour. $\mathrm{Zn}$ atoms are represented as grey spheres at each active-site. (b) Molecular surface of the BHMT tetramer showing the interface between the different subunits. In addition to the close interaction between monomers within each dimer (AB and $C D)$, the dimers are bound together by the long $\mathrm{C}$-terminal helixes, which are anchored to a different subunit, thus enlarging the interface between them.

entrance to the active-site cavity. Loop L2 (residues 79-95), on the contrary, extends away from the barrel, its great mobility playing an important role in homocysteine binding, as shown below. As it can also be seen in Figure 2(b), the C-terminal (a)
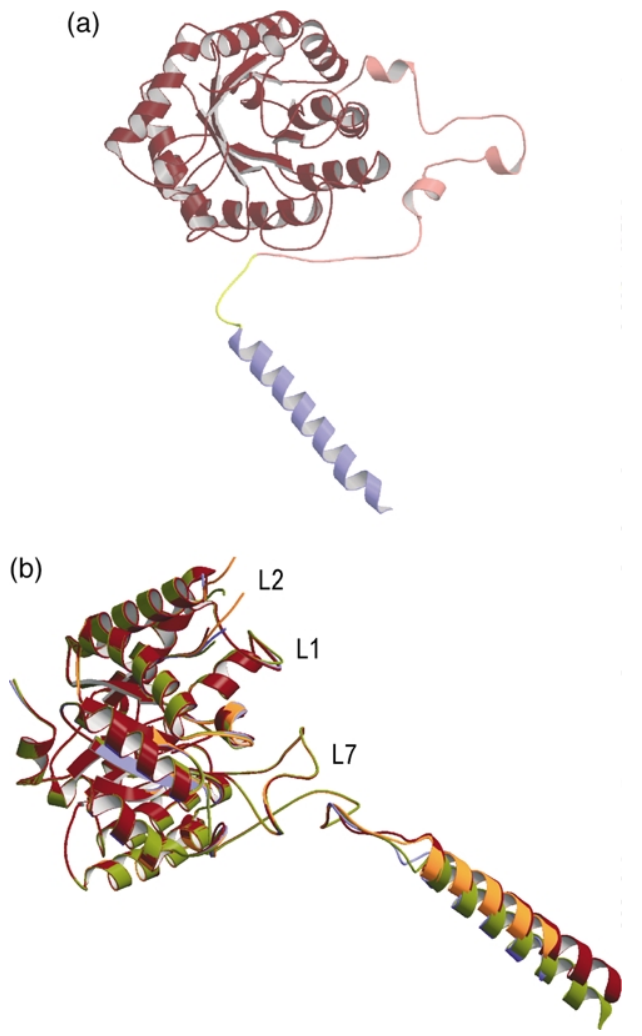

Figure 2. The protomer of rat liver BHMT. (a) The overall fold consists of a $(\alpha / \beta)_{8}$ barrel including residues 10-318 (red), an extended arm including residues 319-370 (pink), a flexible linker from 371 to 380 (yellow), and a long $\alpha$-helix including residues 381-406 (purple). The extended arm is closely interacting with the other subunit within the dimer and also with the long C-terminal helix from the other dimer. (b) View perpendicular to (a). The four monomers are superimposed showing that the helices are flexible and similarly oriented in pairs $\mathrm{AD}$ and $\mathrm{BC}$, with a tilt of $11^{\circ}$ between them. The L1 and L7 loops from the barrel lie over the active site, whereas L2 is extended and presents a great mobility playing an essential role in Hcy binding.

helix is flexible and presents different orientation in monomers $\mathrm{A}$ and $\mathrm{D}$ with respect to the other two $(B, C)$, the tilt being $11^{\circ}$ between both pairs and the linker loop 372-380 acting as the hinge. This different orientation of the C-terminal helix is most probably reflecting the different crystal packing environment of the four subunits. In any case, the overall folding of the subunit is similar to that described for the human enzyme but the long extra helix, which was not observed in the human crystals, is protruding into the opposite dimer and, therefore, both dimers are interacting to a greater extent than reported.

\section{The subunits interaction in the tetramer}

Although preliminary experiments presumed the existence of hexameric BHMT, the tetrameric aggregation had already been accepted, ${ }^{15}$ and the human enzyme structure confirmed this 
assumption. $^{21}$ However, the weak interface between dimers as found in those crystals prevented the exclusion of other modes of aggregation in solution. Nevertheless, this structure was incomplete at the C-terminal region (residues 373-406). On the other hand, the secondary structure prediction methods clearly assign a long $\alpha$-helix from residue 380 to the $C$ terminus of the polypeptide chain. ${ }^{20}$ However, this segment could not be built in the model we reported ${ }^{20}$ as it was not contained in the template used, and in any case, this segment was not shared by all the members of the thiol-selenol methyltransferases family. This last observation led to the assumption that the C-terminal extension found in BHMT is not necessary for folding.

From residue 318, at the end of helix 8, the polypeptide chain extends away from the barrel in the so called dimerization arm, making many inter- actions with the other subunit. ${ }^{21}$ In particular, a long loop is defined by residues 332-348 from molecule A that interacts closely with L1 and L7 loops from monomer $\mathrm{B}$, and contributes in shaping the entrance to the active-site cavity of this last subunit located opposite to A (see Figure 1(b)). From here, chain $\mathrm{A}$ is interacting with the equivalent segment from monomer $\mathrm{B}$, and defines $\mathrm{a}$ motif, so named as the "hook", which enlarges the subunits interface within the dimer. However, the structure of rat liver BHMT herein presented shows that this extended arm is involved in dimerization and in the stabilization of the tetramer. The segment 316-349 of chain A, at the beginning of this long extended arm, is shaping a crevice over the molecular surface (Figure 1(b)). The electron density maps of rat liver BHMT show a clear density in this crevice, corresponding to a helix coming from molecule $\mathrm{C}$, situated below. The

(a)

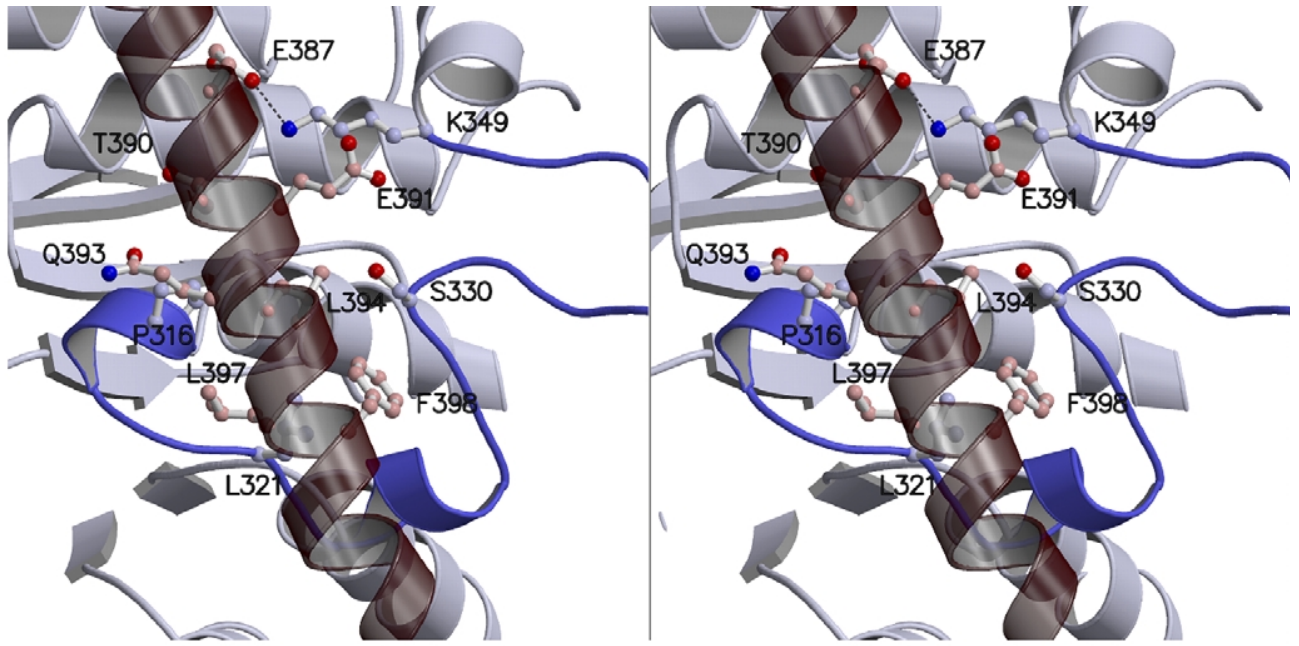

(b)
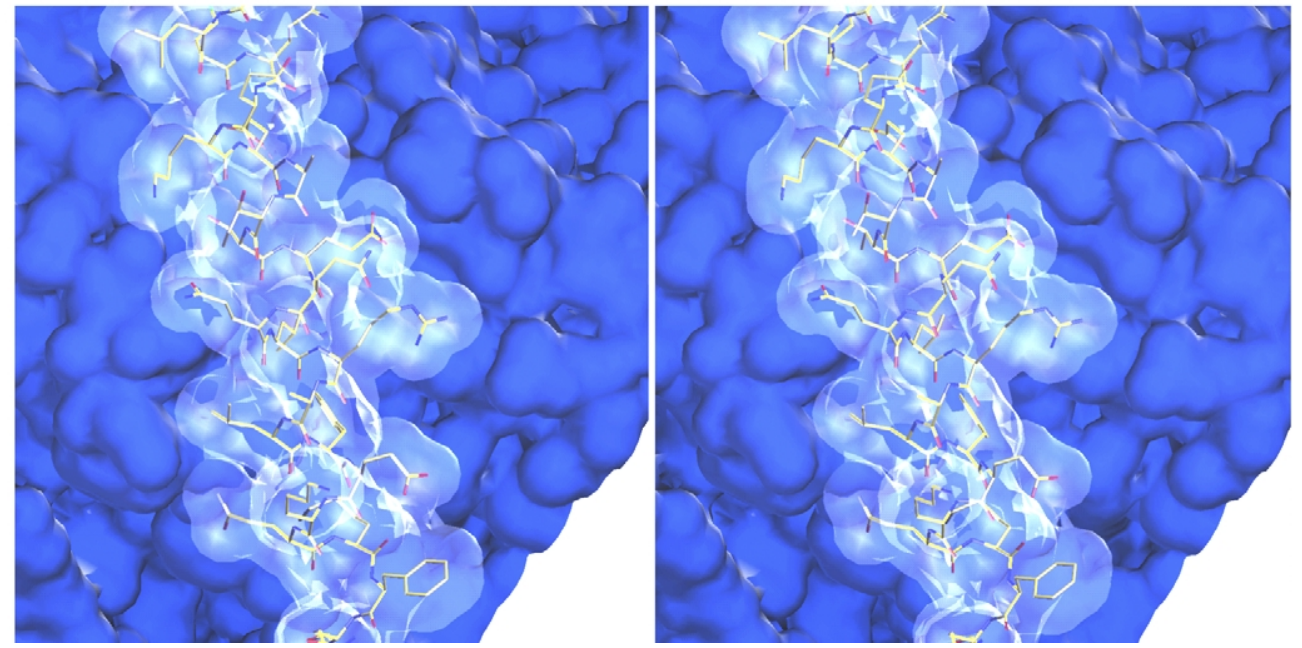

Figure 3. The C-terminal helix. (a) A detail showing the atomic interactions between the segment Pro316-Lys349 (dark blue) of the extended arm from molecule D and the helix (transparent red) from molecule B, in the same view that is shown in Figure 1. In addition to the Glu387-Lys349 ion-pair, more than 60 hydrophobic interactions are produced, the main residues involved being displayed and labelled. (b) Molecular surface at the interface, the helix being transparent and filled with the corresponding residues as sticks. Thr390, Leu394 and Phe398 from the helix are buried in a very narrow hydrophobic pocket. The remaining residues, such as Glu391, Gln393 and Leu397, are fitting their side-chain to the molecular surface, enlarging the interface and burying a total of about $1500 \AA^{2}$. 
C-terminal helix of molecule $\mathrm{A}$ is, in turn, anchored to the surface defined by the dimerization arm of molecule C (which is hidden in Figure 1(b)). Besides, the same interaction is formed between molecules B and D, and therefore, the four long $\mathrm{C}$-terminal helices bind together the tight dimers $\mathrm{AB}$ and $\mathrm{CD}$.

As described above, this helix is closely packed with the segment Pro316-Lys349 of the polypeptide chain from another subunit. A detail of the helix represented in Figure 1(b) is shown in Figure 3(a). There is a strong ion-pair between Glu387(OE2), from the helix, and Lys349(NH) at the end of this loop. From this Glu387 to the end, more than 60 hydrophobic interactions (less than $4 \AA$ ) are produced between the buried part of the helix and several residues of the loop, about 15 of these contacts being closer than $3.5 \AA$ (Figure 3(a)). In particular, the side-chain of Thr390, Leu394 and Phe398 from the helix are buried in a very narrow hydrophobic pocket shaped mostly by Pro316, Leu321 and the main-chain from Gly329 and Ser330. The remaining residues, as Glu391, Gln393 and Leu397, are fitting their side-chain to the molecular surface through hydrophobic interactions, enlarging the interface and burying a total of about $1500 \mathrm{~A}^{2}$ (Figure 3(b)). Therefore, although this segment of the chain seems not to be crucial for proper folding of the subunit, it does stabilize the tetramer association between the monomers by protruding deeply into the molecular surface of the opposite dimer.
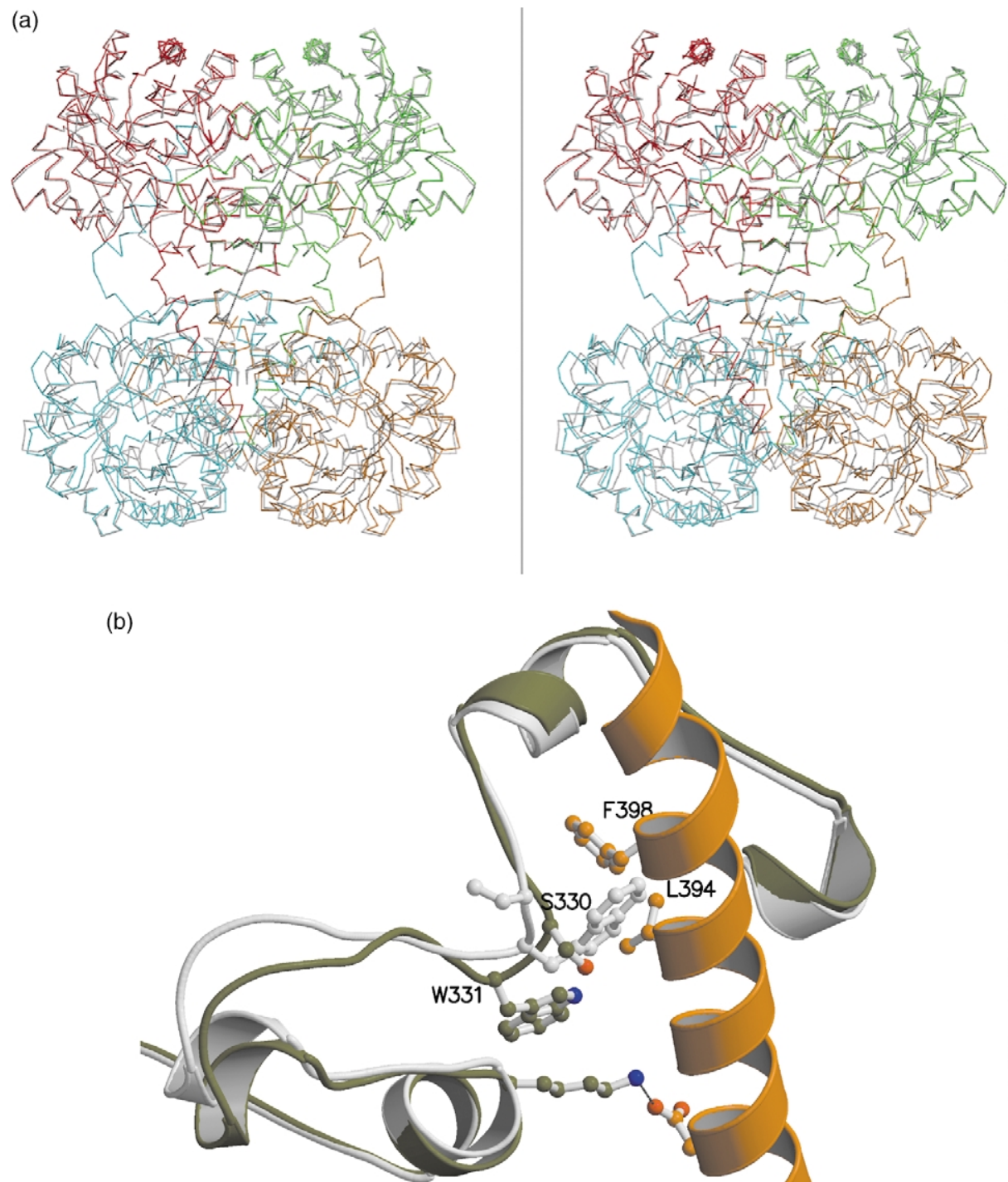

Figure 4. BHMT versus h-BHMT tetramer. (a) Superposition of dimers (AB) from human (grey) and rat liver (red and green) BHMT, giving a RMS of $0.91 \AA$. An $8^{\circ}$ rotation of the CD dimer (blue and orange units) about the represented axis is needed to optimally superimpose to the h-BHMT tetramer. (b) A detail showing the interaction between molecule A and helix-C. The main-chain of A is flipped at the Ser339-Trp331 bond, and Trp331 in h-BHMT is occupying the narrow hydrophobic pocket filled by Leu394 and Phe397 from the helix-C in rat liver BHMT. This has the effect of smoothing the crevice at the interface of the human enzyme. This change is observed in the dimerization arms from A and D, both at the same face of the tetramer, but not in the other two. 


\section{Comparison with human BHMT}

The overall architecture of the tetramer is the same as that found in h-BHMT, but the arrangement of the subunits is slightly different, as can be seen in Figure 4(a), where the dimers AB from each enzyme are superimposed. These dimers are quite conserved (RMS $0.91 \AA$ ) but an $8^{\circ}$ rotation of the $\mathrm{CD}$ dimer is needed to superimpose optimally to the h-BHMT tetramer. Furthermore, a careful inspection of the $A B$ dimers shows interesting differences in the extended arm from subunit $\mathrm{A}$ in both enzymes, a zoom of this region being detailed in Figure 4(b). The chain is flipped in the molecule A along the Ser330-Trp331 bond, and the sidechain of this Trp331 of h-BHMT is tilted with respect to the rat liver enzyme filling the narrow hydrophobic pocket occupied by Leu394 and Phe397 from the helix-C in rat liver BHMT. This has the effect of smoothing the crevice at the interface of the human enzyme, which, therefore, could not accommodate the helix. Interestingly this fact is observed in the dimerization arm of molecules A and D, both at the same face of the tetramer, but not in $B$ and $C$. Thus, on the other face of the human tetramer, the molecular surface shaped by $B$ and $C$ shows two crevices that could possibly be occupied by helices from molecules D and A, respectively, which appear disordered in the crystals from human BHMT. Consequently with this observation, the crystal packing for h-BHMT shows room to accommodate these two putative helices.

\section{Active site and the $\mathrm{Zn}$ coordination}

Several studies directed to establish a structural and/or catalytic role for $\mathrm{Zn}$ in proteins have been reported. ${ }^{22-25} \mathrm{Zn}$ fingers are the most typical motifs with a structural role for the $\mathrm{Zn}$, the metal being tetrahedrically coordinated to His and/or Cys residues. On the other hand, catalytic roles for $\mathrm{Zn}$ in proteins include the activation of water for nucleophylic attack and the electrophylic activation of certain groups, as the aldehyde carbonyl group in alcohol dehydrogenase. In this case, the most common ligand is His, followed by Asp, $\mathrm{Glu}$, and $\mathrm{Cys}^{22}$ and the preferred coordination is tetrahedral, although there are cases in which $\mathrm{Zn}$ is penta-coordinated, since this metal is capable of changing its coordination number.

A new role for $\mathrm{Zn}$ has emerged in the last few years as activator of thiols, as is the case in BHMT and the thiol/selenol methyltransferases family. ${ }^{26}$ The reaction catalysed by BHMT involves a nucleophylic attack from Hcy to one of the betaine methyl groups, generating methionine plus dimethylglycine (see Figure 5(a)). The nucleophylic activation of homocysteine is reached upon coordination to the $\mathrm{Zn}$ atom. It has been suggested that coordination of the substrate thiol to the $\mathrm{Zn}^{2+}$ lowers the $\mathrm{p} K_{\mathrm{a}}$ of the thiol, thereby increasing the concentration of thiolate at neutral $\mathrm{pH}$, and there-

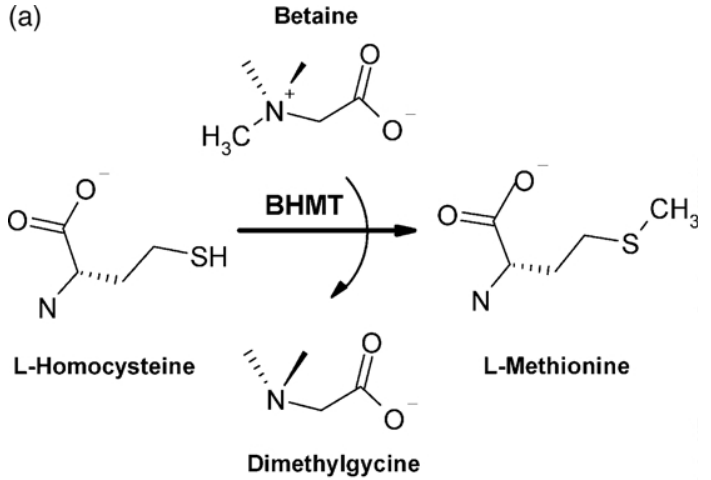

(b)

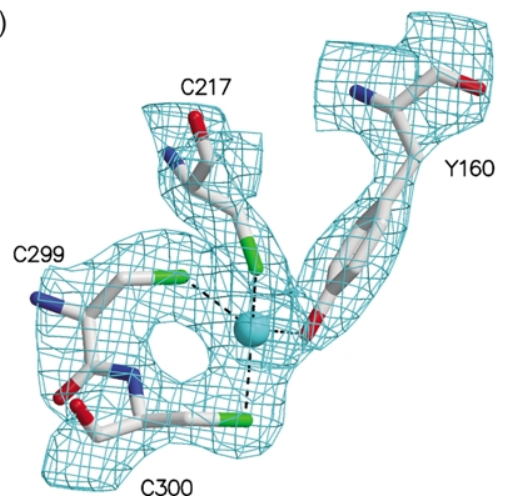

Figure 5. The role of $\mathrm{Zn}$ as activator of thiols (a) The enzymatic reaction catalyzed by BHMT. (b) Final $2 F_{\mathrm{o}}-F_{\mathrm{c}}$ electron density map contoured at the $1 \sigma$ level, showing the three essential Cys residues and Tyr160 coordinated to the $\mathrm{Zn}$ atom. Tyr is conserved in the BHMT enzymes, the other members of the thiol-selenol methyltransferases having Thr at this position.

fore activating the thiol for methyl transfer under physiological conditions. This role of $\mathrm{Zn}$ as an activator of thiols to produce thiolates has also been proposed for other proteins as Ada and prenyl transferases. ${ }^{26}$ Many studies have been carried out to determine the Zn ligands of BHMT and the proteins belonging to its family. In particular, EXAFS spectroscopy on B12-MS ${ }^{27,28}$ reveal the existence of three Cys residues and a fourth ligand that would provide an oxygen or nitrogen replaced by homocysteine during catalysis, as the $\mathrm{Zn}$ site appears to remain four-coordinated after the addition of Hcy. BHMT also conserves these three Cys residues (Cys217, Cys299 and Cys300) but there is no clue about which could be the fourth $\mathrm{Zn}$-coordinating residue for any of the enzymes, in spite of the mutagenesis studies done to identify it. $^{18}$

The crystal structure of native rat liver BHMT presented here clearly shows that Tyr160, at the end of the $\beta 4$ strand, is coordinating to the $\mathrm{Zn}$ as the fourth ligand (Figure 5(b)). Furthermore, mutagenesis studies confirm a key role for the phenol group as none of the mutants (Tyr160Ala, Tyr160Phe and Tyr160Thr) show significant BHMT activity (Table 1), all of them presenting the same structural features as the native. Alignment between BHMT family shows that Tyr160 is a 
conserved residue in all of them. On the contrary, this Tyr is not conserved within the whole thiol/ selenol methyltransferases family, Thr being the most occurring residue at this position, as is the case for B12-MS, the most related enzyme to BHMT. There are only three exceptions that contain Ala: S-homocysteine methyltransferase from Zea mays (mayze) (Q9FUNO) and from Oryza sativa (Q8H825), and selenocysteine methyltransferase from Astragalus bisulcatus (P56707). Nonetheless, the function has been inferred for both Hcys MTs, and, interestingly, there are three more sequences deposited for Zea mays which conserve Thr in this position (Q9FUM7, Q9FUM8 and Q9FUM10). On the other hand, SeCys MT uses SAM as methyl donor and a selenol substrate instead of thiol, which could be responsible for the differences encountered. Noteworthy, only the enzyme from A. bisulcatus has been reported to present Ala at this position, the other reported SeCys MT having $\mathrm{Thr}^{29}$

As mentioned, Asp, Glu, His and Cys are the most common $\mathrm{Zn}$ ligands. However, there are some cases in which Thr, Tyr, and Ser play such a role. For instance, Astacin and the Zn-proteases family related to $\mathrm{it}^{30}$ present three $\mathrm{His}$ residues and one Tyr, whereas one Cys, two His residues and one Thr coordinate the $\mathrm{Zn}$ atom in threonyltRNA synthetase. ${ }^{31}$ In this sense, it is remarkable that the strong inhibition of human BHMT by phosphinic pseudo peptide compounds has been recently reported. ${ }^{32}$ In this work, it is assumed that the inhibitor interacts with the Zn via its phosphinate group by competing with Hcy for the metal, as the interaction is reversed using Hcy but not betaine. Interestingly, the direct evidence of interaction between $\mathrm{Zn}$ and oxygen from phosphinate group has been proved by the crystal structure of the complex between Astacin and a phosphinate inhibitor, ${ }^{33}$ in which the Tyr ligand is replaced by the phosphinate. Therefore, BHMT seems to display a similar inhibition mechanism, the phosphinate competing with Hcy in replacing the Tyr coordinated to the $\mathrm{Zn}$ atom.

\section{Recognition and binding of substrates}

Crystallization of human BHMT in the presence of a transition state analogue (CB-Hcy) allowed the characterization of the residues involved in substrate recognition. ${ }^{21}$ Essentially, the determinants responsible for Hcy binding are the mainchain at Phe29-Val30, providing hydrogen bonds to the Hcy-COOH group, and the Glu159, providing stereoespecifity in binding the NH2. For betaine, several amino acid residues (Tyr77, Tyr160, Phe261 and Phe267) create a hydrophobic environment, which presumably would protect the betaine methyl groups. Besides, Trp44 and Tyr77 interact with betaine carboxylates through their NE1 and $\mathrm{OH}$ atoms, respectively.

The structure of free BHMT reveals some interesting differences as compared to the complexed form. Figure 6 shows that a change in Tyr160 sidechain conformation is needed to allow the entrance of the substrate. It is assumed that the $\mathrm{SH}-$ group of Hcy replaces the $\mathrm{OH}$ - group of Tyr, which moves apart to a new position. Interestingly, this new position helps in shaping the hydrophobic core for betaine. This kind of "Tyr switch" upon substrate binding has been described in Astacin ${ }^{30}$ and in other proteins of the Astacyn family as Serratia and Pseudomona proteases. ${ }^{34,35}$ Nevertheless, while mutations on Tyr160 bring about a dramatic effect on enzymatic activity, the mutation of this Tyr in Astacin presents a more moderate effect. $^{36}$ This is consistent with a double role of Tyr in BHMT activity, both as $\mathrm{Zn}$ ligand replaced by Hcy upon binding, and also as part of the betaine site. Besides, this second role for Tyr160 would justify that other enzymes of the family do not conserve this residue, as they use a different methyl
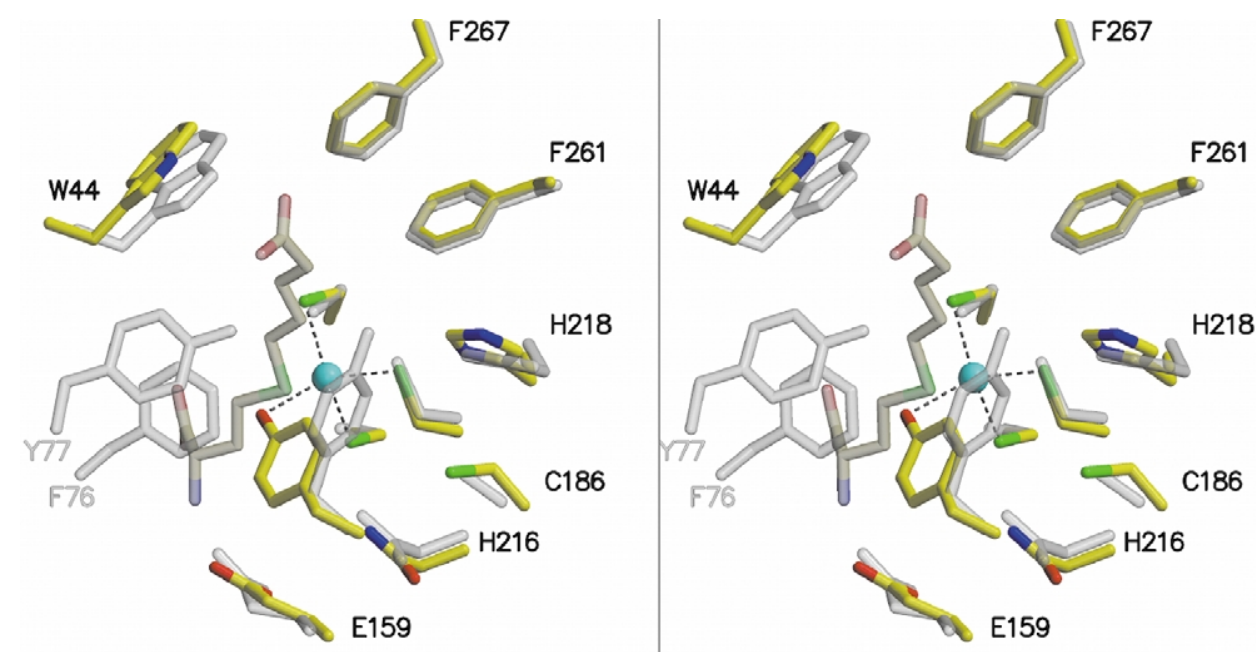

Figure 6. The active site of free and bound BHMTs: comparison of rat liver BHMT and the complex of h-BHMT with the CB-Hcy transition-state analogue (white). The entrance of Hcy, stacked to Phe76, switches Tyr160 to a new position which contributes in shaping the betaine-binding site. 
donor source. Thus, the presence of Thr at this position enables the coordination to $\mathrm{Zn}$, and the displacement by Hcy. But the transfer mechanism must display differences within the family. For example in the case of B12-MS, different domains of the protein bind the substrate Hcy, the methyl donor, methyltetrahydrofolate and even its cofactor, cobalamine. ${ }^{37}$

Furthermore, the comparison of both structures reveals a much more observable effect in the conformation of L2, the loop emerging from $\beta 2$ of the barrel (Figure 7). This loop is partially disordered in both BHMT enzymes, Ser79 being the last residue defined in the electron density maps from rat liver BHMT crystals. However, the visible portion of the loop in the free and complexed forms is very different, revealing a significant conformational change upon binding of the substrates. When the active site is empty, this loop remains extended and out of the active site, but when CB-
HCy occupies the active site, part of this loop moves towards the centre of the barrel providing two residues (Phe76 and Tyr77) which are essential for the binding of the substrates. Phe76 has been included in the betaine-binding site, but we think it is mainly involved in properly orienting the Hcy by interaction with its hydrophobic moiety. BHMT and B12-MS both conserve a Phe in this position, whereas a Tyr is found in the other members of the family. Therefore, the presence of an aromatic residue at this position seems essential for Hcy recognition and binding. On the other hand, Tyr77 is contributing to the hydrophobic core for betaine, and also provides a hydrogen bond for the betaine carboxylate. Thus, it seems that at first, Phe76 is involved in Hcy binding, and then, it could trigger the conformational change after which the betainebinding site is fully created. This assumption matches perfectly with the kinetics of BHMT that shows an ordered reaction, Hcy binding first. To
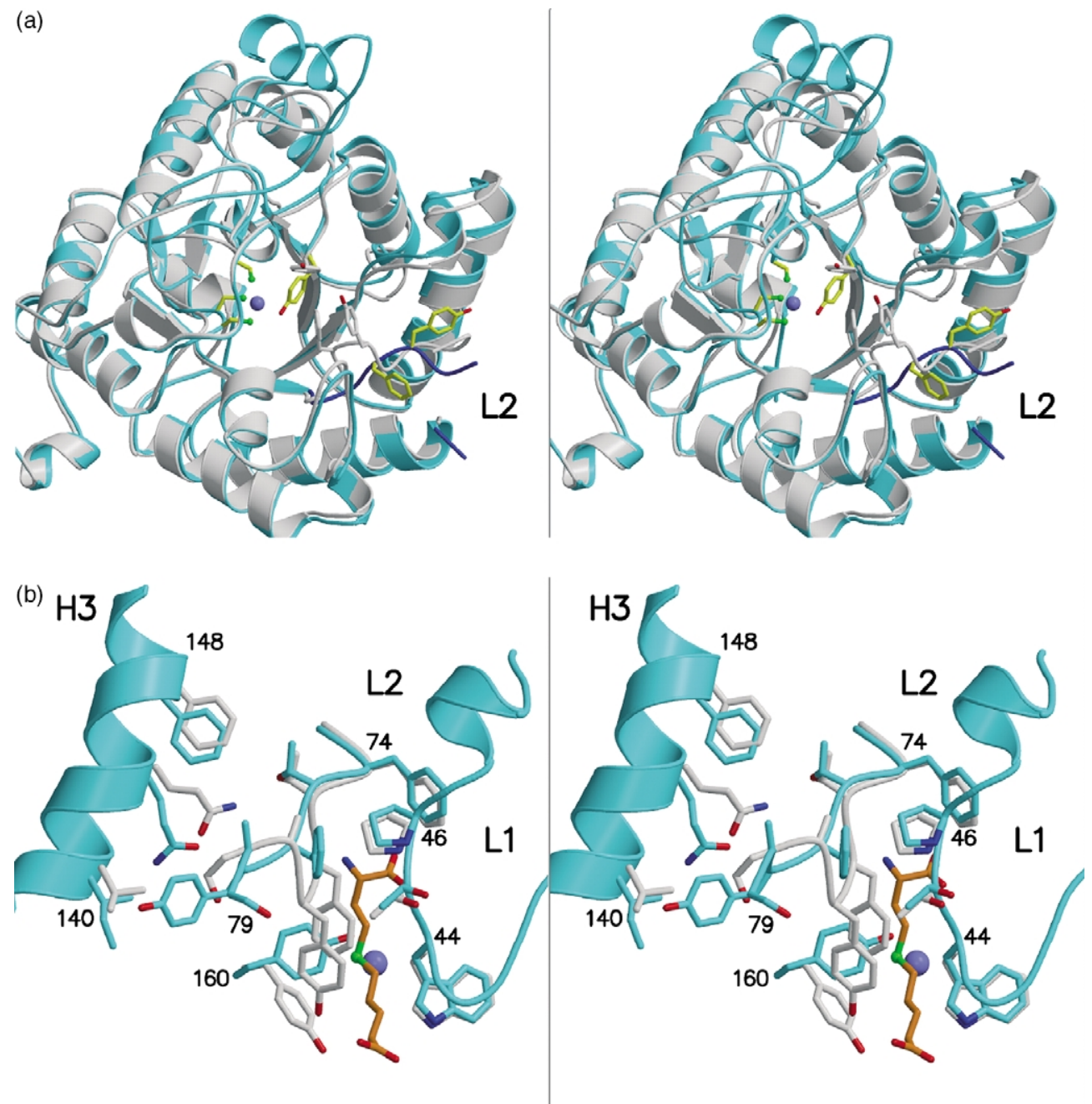

Figure 7. Conformational change upon substrate binding. (a) The subunit from rat liver BHMT (cyan) is superimposed to the complexed monomer from h-BHMT (white) showing the movement on the L2 loop (dark blue in rat liver), which is extended in the free enzyme and that moves toward the active site upon substrates binding, providing the essential Phe76 and Tyr77 residues. This, in turn, switches Tyr160 from the Zn-coordinated position. (b) A detail of the atomic interaction of L2 residues in the segment Phe74-Ser79. Phe76 is closely interacting with Thr45 and Pro46 from the L1 loop of free BHMT, while Tyr77 is making hydrophobic contact to Ile140 from helix H3. 
confirm the essential role of these residues we have carried out mutagenesis studies on both positions, the mutants of Phe76 to Ala and Asp showing no BHMT enzymatic activity. Mutation on Tyr77 described (Tyr77Ala) ${ }^{20}$ reduces about tenfold BHMT activity. This residue would be less critical than Phe76, since the main conformational change would be produced before, and Tyr77 would be involved in the subsequent binding of the second substrate, betaine. Consistently with this role, Tyr is specific for the BHMT enzymes.

Conformational changes have been predicted based on alterations in fluorescent spectra due to binding of both substrates. ${ }^{21}$ These changes have been attributed to Trp44 of L1 loop (residues 3852) which is disordered in the unbound human enzyme but ordered in the complex. However, our results show that L1 is perfectly ordered in the free-state and no changes in its conformation should be expected to accommodate the substrates (Figure 7(b)). On the contrary, the change in the L2 loop conformation upon substrates binding must display observable variations in the spectra, due to the significant change in the Trp44 environment.

A last point worth mentioning is the proximity of the Cys186 to the catalytic Zn (Figure 6). This residue, mutated in human BHMT crystals to Ala, is very close to the essential Cys217, but also to Asn216 and His218, both forming part of the second coordination sphere of the metal by providing H-bonds to Cys217 and Cys300. Measurements of methionine synthesis carried out on Cys186Ala and Cys186Ser mutants showed that although it is not critical for enzyme activity, it does change the affinity for the substrates, especially for Hcy. ${ }^{20}$ The reason for this effect is not clear, but could be related to a stabilizing interaction of Cys186 with the switched position of Tyr160, as both residues, Tyr and Cys, are specific for the BHMT enzymes.

In summary, the structure of rat liver BHMT herein presented shows many new interesting features, as the existence of a C-terminal $\alpha$-helix that contributes to increase the interface between subunits, thus enhancing the stability of the oligomer. This same secondary structure element, as well as a similar pattern of interactions is expected to occur in human BHMT due to the high level of identity observed with the rat sequence. Our work also shows the evidence that Tyr160 is the fourth ligand coordinated to the $\mathrm{Zn}$ and substituted by Hcy in the BHMT enzymes. A hydroxyl group bound to the $\mathrm{Zn}$ seems to be a common feature of the thiol-selenol methyltransferases family, and this reveals a similar mechanism in activating the substrate. However, the presence of a Thr in other members of the family reflects the diversity in the molecules that provide the methyl group. Thus, there must be structural requirements which are specific for the different functionality within the family. Nevertheless, many details of the methyl transfer process remain unknown and more work needs to be done in order to fully understand the enzymatic mechanism of these enzymes.

\section{Materials and Methods}

\section{Expression and purification of rat liver BHMT}

Expression and purification of rat liver BHMT was carried out using the pET11a-BHMT plasmid as described. ${ }^{15}$ The protein was characterized by gel filtration chromatography, analytical ultracentrifugation and $C D$ spectroscopy and found to preserve the main features of the liver purified enzyme. ${ }^{20}$ BHMT activity measurements were carried out for one hour at $37^{\circ} \mathrm{C}$ using the radio assay described. ${ }^{38}$

\section{Site-directed mutagenesis, expression, purification and characterization of the rat liver BHMT mutants}

Site-directed mutagenesis was carried out using the plasmid pTYB12-BHMT as described ${ }^{20}$ and the following mutagenic oligonucleotides (sense strand shown; the substitutions appear in bold):

Y160F, 5'-GGACTTCCTCATTGCAGAGTTTTTTG AACATGTTG-3';

Y160T, 5'-GGACTTCCTCATTGCAGAGACTTTTG AACATGTTG-3';

Y160A, 5'-GGACTTCCTCATTGCAGAGGCTTTTG AACATGTTG-3';

F76A， 5'-GCAGACCTTCACTGCCTATGCAAGT GAGG-3';

F76D, 5'-GCAGACCTTCACTGACTATGCAAGTG AGGAC-3'.

Mutations were verified by sequencing the entire cDNA, using the dideoxy termination method. ${ }^{39}$ Expression, purification and characterization of the mutants was performed as described. ${ }^{20}$ None of them showed significant changes in the CD-spectra with respect to the native protein. For storage purified BHMT was made $50 \%(\mathrm{v} / \mathrm{v})$ glycerol and kept at $-20{ }^{\circ} \mathrm{C}$.

\section{Crystallization and data collection}

The crystallization of rat liver BHMT has been described. ${ }^{15}$ Equal amounts of protein solution $(6 \mathrm{mg} / \mathrm{ml}$ BHMT in $10 \mathrm{mM}$ Tris $-\mathrm{HCl}(\mathrm{pH} 8.2), 0.5 \mathrm{mM}$ EDTA and $1 \mathrm{mM}$ 2-mercaptoethanol) and precipitant buffer $(14 \%$ (w/v) PEG 2K MME in $100 \mathrm{mM}$ Tris-HCl, $\mathrm{pH}$ 8.5) were mixed and vapour equilibrated against a reservoir containing the latter solution. Crystallization experiments were carried out at room temperature. Plate-like crystals were very thin, most of them grown as twinned clusters. Crystals, of maximum size $0.2 \mathrm{~mm} \times 0.1 \mathrm{~mm} \times 0.05 \mathrm{~mm}$, belong to $P 2_{1}$ space-group, with four molecules per asymmetric unit and $45 \%$ solvent content in the cell. For data collection, two native crystals were soaked in cryoprotectant solutions containing the precipitant and $20 \%$ glycerol before being cooled to $100 \mathrm{~K}$. Diffraction data were collected using synchrotron radiation at BM14 beam line of the ESRF station at Grenoble (France). The data were processed using Mosflm $^{40}$ and merged with the CCP4 package ${ }^{41}$ to yield integrated intensities. A summary of data collection and data refinement statistics is shown in Table 2. 
Table 2. Data collection and refinement statistics

\begin{tabular}{|c|c|}
\hline \multicolumn{2}{|l|}{ A. Data collection } \\
\hline Temperature (K) & 120 \\
\hline Source & Synchrotron (ESRF) \\
\hline Space group & $P 2_{1}$ \\
\hline Unit cell dimensions $(\AA$, deg. $)$ & $57.83,149.27,96.25, \beta=92.92$ \\
\hline Unique reflections & 58,532 \\
\hline$R_{\text {sym }}$ & $0.088(0.40)$ \\
\hline Completeness (\%) & $99.8(99.7)$ \\
\hline Mean multiplicity & $4.0(4.0)$ \\
\hline Mean $I / \sigma I$ & $6.5(1.5)$ \\
\hline \multicolumn{2}{|l|}{ B. Final refinement parameters } \\
\hline Resolution range $(\AA)$ & $48.4-2.5$ \\
\hline \multicolumn{2}{|l|}{ Number of reflexions } \\
\hline Working set & 54,654 \\
\hline Test set & 5548 \\
\hline Protein atoms (non-H) & 11,807 \\
\hline Heterogen atoms (non-H) & 8 \\
\hline Solvent molecules & 547 \\
\hline$R$-factor & 0.23 \\
\hline$R_{\text {free }}$ & 0.28 \\
\hline \multicolumn{2}{|l|}{ RMSD } \\
\hline Bond lengths ( & 0.009 \\
\hline Bond angles (deg.) & 1.4 \\
\hline Dihedral angles (deg.) & 22.1 \\
\hline Improper angles (deg.) & 0.93 \\
\hline \multicolumn{2}{|l|}{ Averaged $B$-factors $\left(\AA^{2}\right)$} \\
\hline Protein & 48.6 \\
\hline Solvent & 47.2 \\
\hline
\end{tabular}

\section{Structure solution and refinement}

As the structure of the human enzyme became available, rat liver BHMT structure was solved by molecular replacement with the AMoRe program, ${ }^{42}$ by using the human monomeric subunit as a search model (PDB code: 1LT8). Four solutions corresponding to the full tetramer were found, which after rigid-body fitting produced an $R$-factor of 0.39 with a correlation coefficient of 0.58 for data up to $3.5 \AA$ resolution. The tetramer presents point symmetry 222 , with non-crystallographic 2 -fold symmetry nearly along the axes $a$ and $c$. Crystallographic refinement was performed by using $\mathrm{CNS}^{43}$ with flat bulk-solvent correction and including low-resolution data to $50 \AA$. A subset of $10 \%$ randomly selected structure-factor amplitudes were excluded from automated refinement to compute a free $R$-factor throughout the refinement. Several rounds of SA-refinement imposing NCS-restraints between the four subunits were alternated with rebuilding by the program $\mathrm{O} .{ }^{44}$ The initial electron-density maps showed a well-defined density that revealed the presence of a long helix packed against both faces of each dimer interface. The traces of these helices were manually built and included in the model. Further cycles of NCS-restrained refinement allowed the identification of most residues in the C-terminal helix and a connected density for the linker segment 371-380. As it was clear that the orientation of this C-terminal helix was slightly different in the four subunits, the linker was excluded from the NCS-restraints, the final model showing density higher than $1 \sigma$ for this segment in all four molecules of the asymmetric unit. Major changes with respect to the human model were also included in the loop connecting $\beta 2$ to $\alpha 2$ of the barrel, which nevertheless is disordered in a variable extent depending on the subunit. No visible density was observed at the $\mathrm{N}$ terminus before Lys10 for any of the subunits, so this portion was not modelled. At the active site, SA-omit maps were computed around the $\mathrm{Zn}$ position to confirm the metal coordination. No restraints were used for the metal-ligand distances during refinement.

At the last stage, water molecules were included combined with more rounds of positional and individual restrained $B$-factor refinement, which led to a final $R$-factor of $0.23\left(R_{\text {free }}=0.28\right)$ for all data sets up to $2.5 \AA$ resolution. Final refinement parameters are reported in Table 2. Stereochemistry of the model was checked by using PROCHECK ${ }^{45}$ and the Figures were generated with MOLSCRIPT, ${ }^{46}$ Raster 3D, ${ }^{47}$ GRASP $^{48}$ and O. ${ }^{44}$

\section{Protein Data Bank accession numbers}

The atomic co-ordinates have been deposited in the RCSB Protein Data Bank (accession codes: 1UMY).

\section{Acknowledgements}

This work has been supported by grants of Instituto de Salud Carlos III (FIS 01/1077 and RCMN C03/08) (M.A.P.), Ministerio de Ciencia y Tecnología (PM 97/0064 and BMC 2002-00243 (M.A.P.) and BIO2000-1279-C02-02 (J.S.A.)). We thank Francisco Garrido for technical assistance, María Gasset for her help with CD spectra, and Brenda Ashley Morris for style and grammatical corrections.

\section{References}

1. Mato, J. M., Alvarez, L., Ortiz, P. \& Pajares, M. A. (1997). S-adenosylmethionine synthesis: molecular mechanisms and clinical implications. Pharmacol. Ther. 73, 265-280.

2. McCully, K. S. (1969). Vascular pathology of homocysteinemia: implications for the pathogenesis of arteriosclerosis. Am. J. Pathol. 56, 111-128.

3. Refsum, H., Ueland, P. M., Nygard, O. \& Vollset, S. E. (1998). Homocysteine and cardiovascular disease. Annu. Rev. Med. 49, 31-62.

4. Barak, A. J. \& Tuma, D. J. (1979). A simplified procedure for the determination of betaine in liver. Lipids, 14, 860-863.

5. García-Pérez, A. \& Burg, M. B. (1991). Renal medullary organic osmolytes. Physiol. Rev. 71, 1081-1115.

6. McCue, K. F. \& Hanson, A. D. (1992). Salt-inducible betaine aldehyde-dehydrogenase from sugar beet: cDNA cloning and expression. Plant Mol. Biol. 18, $1-11$.

7. Delgado-Reyes, C. V., Wallig, M. A. \& Garrow, T. A. (2001). Immunohistochemical detection of betainehomocysteine S-methyltransferase in human, pig, and rat liver and kidney. Arch. Biochem. Biophys. 393, 184-186.

8. Rao, P. V., Garrow, T. A., John, F., Garland, D., Millian, N. S. \& Zigler, J. S., Jr (1998). Betaine-homocysteine methyltransferase is a developmentally regulated enzyme crystallin in rhesus monkey lens. J. Biol. Chem. 273, 30669-30674.

9. Sandu, C., Nick, P., Hess, D., Schiltz, E., Garrow, T. A. \& Brandsch, R. (2000). Association of betaine-homocysteine S-methyltransferase with microtubules. Biol. Chem. 381, 619-622. 
10. Furuya, N., Kanazawa, T., Fujimura, S., Ueno, T., Kominami, E. \& Kadowaki, M. (2001). Leupeptininduced appearance of partial fragment of betaine homocysteine methyltransferase during autophagic maturation in rat hepatocytes. J. Biochem. 129, 313-320.

11. Sowden, M. P., Collins, H. L., Smith, H. C., Garrow, T. A., Sparks, J. D. \& Sparks, C. E. (1999). Apolipoprotein B mRNA and lipoprotein secretion are increased in McArdle RH-7777 cells by expression of betaine-homocysteine S-methyltransferase. Biochem. J. 341, 639-645.

12. Forestier, M., Reichen, J. \& Solioz, M. (1996). Application of mRNA differential display to liver cirrhosis: reduced fetuin expression in biliary cirrhosis in the rat. Biochem. Biophys. Res. Commun. 225, 377-383.

13. Garrow, T. A. (1996). Purification, kinetic properties, and cDNA cloning of mammalian betaine-homocysteine methyltransferase. J. Biol. Chem. 271, 22831-22838.

14. Bose, N. \& Momany, C. (2001). Crystallization and preliminary X-ray crystallographic studies of recombinant human betaine-homocysteine S-methyltransferase. Acta Crystallog. sect. D, 57, 431-433.

15. González, B., Pajares, M. A., Too, H.-P., Garrido, F., Blundell, T. L. \& Sanz-Aparicio, J. (2002). Crystallization and preliminary X-ray study of recombinant betaine-homocysteine S-methyltransferase from rat liver. Acta Crystallog. sect. D, 58, 1507-1510.

16. Millian, N. S. \& Garrow, T. A. (1998). Human betaine-homocysteine methyltransferase is a zinc metalloenzyme. Arch. Biochem. Biophys. 356, 93-98.

17. Breksa, A. P., III \& Garrow, T. A. (1999). Recombinant human liver betaine-homocysteine S-methyltransferase: identification of three cysteine residues critical for zinc binding. Biochemistry, 38, 13991-13998.

18. Heil, S. G., Lievers, K. J., Boers, G. H., Verhoef, P., den Heijer, M., Trijbels, F. J. \& Blom, H. J. (2000). Betaine-homocysteine methyltransferase (BHMT): genomic sequencing and relevance to hyperhomocysteinemia and vascular disease in humans. Mol. Genet. Metab. 71, 511-519.

19. Breksa, A. P., III \& Garrow, T. A. (2002). Random mutagenesis of the zinc-binding motif of betainehomocysteine methyltransferase reveals that Gly214 is essential. Arch. Biochem. Biophys. 399, 73-80.

20. González, B., Campillo, N., Garrido, F., Gasset, M., Sanz-Aparicio, J. \& Pajares, M. A. (2003). Active-site mutagenesis study of rat liver betaine homocysteine S-methyltransferase. Biochem. J. 370, 945-952.

21. Evans, J. C., Huddler, D. P., Jiracek, J., Castro, C., Millian, N. S., Garrow, T. A. \& Ludwig, M. L. (2002). Betaine-homocysteine methyltransferase; zinc in a distorted barrel. Structure, 10, 1159-1171.

22. Vallee, B. L. \& Auld, D. S. (1990). Zinc coordination, function and structure of zinc enzymes and other proteins. Biochemistry, 29, 5647-5659.

23. Vallee, B. L. \& Auld, D. S. (1993). New perspective in zinc biochemistry: cocatalytic sites in multi-enzymes. Biochemistry, 32, 6493-6500.

24. Alberts, I. L., Nadassy, K. \& Wodak, S. J. (1998). Analysis of zinc binding sites in protein structures. Protein Sci. 7, 1700-1716.

25. Dudev, T. \& Lim, C. (2000). Tetrahedral vs. octahedral zinc complexes with ligands of biological interest: a DFT/CDM study. J. Am. Chem. Soc. 122, 11146-11153.

26. Matthews, R. G. \& Goulding, C. W. (1997). Enzyme- catalyzed methyl transfers to thiols: the role of zinc. Curr. Opin. Chem. Biol. 3, 332-339.

27. Peariso, K., Goulding, C. W., Huang, S., Matthews, R. G. \& Penner-Hahn, J. E. (1998). Characterization of the zinc binding site in methionine synthase enzymes of Escherichia coli: the role of zinc in the methylation of homocysteine. J. Am. Chem. Soc. 120, 8410-8416.

28. Peariso, K., Zhou, Z. S., Smith, A. E., Mattthews, R. G. \& Penner-Hahn, E. (2001). Characterization of the $\mathrm{Zn}$ sites in cobalamin-independent and cobalamindependent methionine synthase using zinc and selenium X-ray absorption spectroscopy. Biochemistry, 40, 987-993.

29. Neuhierl, B., Thanbichler, M., Lottspeich, F. \& Böck, A. (1999). A family of S-methylmethionine-dependent thiol/selenol methyltransferases. J. Biol. Chem. 274, 5407-5414.

30. Park, H. I. \& Ming, L.-J. (1998). The mechanistic role of the coordinate tyrosine in astacin. J. Inorg. Chem. $72,57-62$.

31. Sankaranarayanan, R., Dock-Bregeon, A. C., Rees, B., Bovee, M., Caillet, J., Romby, P. et al. (2000). Zinc ion mediated amino acid discrimination by threonyltRNA synthetase. Nature Struct. Biol. 7, 461-4655.

32. Collinsová, M., Castro, C., Garrow, T. A., Yiotakis, A., Dive, V. \& Jirácek, J. (2003). Combining combinatorial chemistry and affinity chromatography: highly selective inhibitors of human betaine:homocysteine S-methyltransferase. Chem. Biol. 10, 113-122.

33. Grams, F., Dive, V., Yiotakis, A., Yiayouros, I., Vassiliou, S., Zwilling, R. et al. (1996). Structure of astacin with a transition-state analogue inhibitor. Nature Struct. Biol. 3, 671-675.

34. Baumann, U., Bauer, M., Letoffe, S., Delepelaire, P. \& Wandersman, C. (1995). Crystal structure of a complex between Serratia marcescens metallo-protease and an inhibitor from Erwinia chrysanthemi. J. Mol. Biol. 248, 653-661.

35. Hamada, K., Hata, Y., Katsuya, Y., Hiramatsu, H., Fujiwara, T. \& Katsube, Y. (1996). Crystal structure of Serratia protease, a zinc-dependent proteinase from Serratia $s p$. E-15 containing a beta-sheet coil motif at $2.0 \AA$ resolution. J. Biochem. 119, 844-851.

36. Yiallouros, I., Berkhoff, E. G. \& Stocker, W. (2000). The roles of Glu93 and Tyr149 in astacin-like zinc peptidases. FEBS Letters, 484, 224-228.

37. Goulding, C. W., Postigo, D. \& Matthews, R. G. (1997). Cobalamin-dependent methionine synthase is a modular protein with distinct regions for binding homocysteine, methyltetrahydrofolate, cobalamin, and adenosylmethionine. Biochemistry, 36, 8082-8091.

38. Finkelstein, J. D. \& Mudd, S. H. (1967). Trans-sulfuration in mammals. The methionine-sparing effect of cystine. J. Biol. Chem. 242, 873-880.

39. Sanger, F., Nicklen, S. \& Coulson, A. R. (1977). DNA sequencing with chain-terminating inhibitors. Proc. Natl Acad. Sci. USA, 74, 5463-5467.

40. Leslie, A. G. W. (1992). Molecular data processing. In Crystallographic Computing 5: From Chemistry to Biology (Moras, D., Podjarny, A. D. \& Thierri, J. C., eds), pp. 39-50, Oxford University Press, Oxford, UK.

41. Collaborative Computational Project Number 4 (1994). The CCP4 suite: programs for protein crystallography. Acta Crystallog. sect. D, 50, 760-763.

42. Navaza, J. (1994). AMoRe: an automated package for 
molecular replacement. Acta Crystallog. sect. A, 50, 157-163.

43. Brünger, A. T., Adams, P. D., Clore, G. M., DeLano, W. L., Gros, P., Grosse-Kunstleve, R. W. et al. (1998). Crystallography \& NMR system: a new software suite for macromolecular structure determination. Acta Crystallog. sect. D, 54, 905-921.

44. Jones, T. A., Zou, J. Y., Cowan, S. W. \& Kjeldgaard, M. (1991). Improved methods for building protein models in electron density maps and the location of errors in these models. Acta Crystallog. sect. A, 47, 110-119.

45. Laskowsky, R. A., MacArthur, M. W., Moss, D. S. \&
Thornton, J. M. (1993). PROCHECK: a program to check the stereochemical quality of protein structures. J. Appl. Crystallog. 26, 283-291.

46. Kraulis, P. J. (1991). MOLSCRIPT: a program to produce both detailed and schematic plots of protein structures. J. Appl. Crystallog. 24, 946-950.

47. Merrit, E. A. \& Bacon, D. J. (1997). Raster3D: photorealistic molecular graphics. Methods Enzymol. 277, 505-524.

48. Nicholls, A. \& Honig, B. A. (1991). Rapid finite difference algorithm utilizing successive overrelaxation to solve the Poisson-Boltzman equation. J. Comp. Chem. 12, 435-445.

Edited by R. Huber

(Received 16 December 2003; received in revised form 3 March 2004; accepted 5 March 2004) 